\title{
Rings of Fractions and Localization
}

\author{
Yasushige Watase \\ Suginami-ku Matsunoki \\ 3-21-6 Tokyo, Japan
}

\begin{abstract}
Summary. This article formalized rings of fractions in the Mizar system [3, 4. A construction of the ring of fractions from an integral domain, namely a quotient field was formalized in 7 .

This article generalizes a construction of fractions to a ring which is commutative and has zero divisor by means of a multiplicatively closed set, say $S$, by known manner. Constructed ring of fraction is denoted by $S^{\sim} R$ instead of $S^{-1} R$ appeared in [1, 6]. As an important example we formalize a ring of fractions by a particular multiplicatively closed set, namely $R \backslash \mathfrak{p}$, where $\mathfrak{p}$ is a prime ideal of $R$. The resulted local ring is denoted by $R_{\mathfrak{p}}$. In our Mizar article it is coded by $R^{\sim} \mathfrak{p}$ as a synonym.

This article contains also the formal proof of a universal property of a ring of fractions, the total-quotient ring, a proof of the equivalence between the totalquotient ring and the quotient field of an integral domain.
\end{abstract}

MSC: 13B30 16S85 68V20

Keywords: rings of fractions; localization; total-quotient ring; quotient field

MML identifier: RINGFRAC, version: 8.1.09 5.60.1371

\section{Preliminaries:}

Units, Zero Divisors and Multiplicatively-Closed Set

From now on $R, R_{1}$ denote commutative rings, $A, B$ denote non degenerated, commutative rings, $o, o_{1}, o_{2}$ denote objects, $r, r_{1}, r_{2}$ denote elements of $R, a$, $a_{1}, a_{2}, b, b_{1}$ denote elements of $A, f$ denotes a function from $R$ into $R_{1}$, and $\mathfrak{p}$ denotes an element of the spectrum of $A$.

Let $R$ be a commutative ring and $r$ be an element of $R$. We say that $r$ is zero-divisible if and only if 
(Def. 1) there exists an element $r_{1}$ of $R$ such that $r_{1} \neq 0_{R}$ and $r \cdot r_{1}=0_{R}$.

Let $A$ be a non degenerated, commutative ring. Let us observe that there exists an element of $A$ which is zero-divisible.

Let us consider $A$.

A zero-divisor of $A$ is a zero-divisible element of $A$. Now we state the propositions:

(1) $0_{A}$ is a zero-divisor of $A$.

(2) $1_{A}$ is not a zero-divisor of $A$.

Let us consider $A$. The functor ZeroDivSet $(A)$ yielding a subset of $A$ is defined by the term

(Def. 2) $\{a$, where $a$ is an element of $A: a$ is a zero-divisor of $A\}$.

The functor NonZeroDivSet $(A)$ yielding a subset of $A$ is defined by the term (Def. 3) $\Omega_{A} \backslash(\operatorname{ZeroDivSet}(A))$.

Let us note that ZeroDivSet $(A)$ is non empty and $\operatorname{NonZeroDivSet}(A)$ is non empty.

Now we state the propositions:

(3) $\quad 0_{A} \notin \operatorname{NonZeroDivSet}(A)$. The theorem is a consequence of (1).

(4) If $A$ is an integral domain, then $\left\{0_{A}\right\}=\operatorname{ZeroDivSet}(A)$. The theorem is a consequence of (1).

(5) $\left\{1_{R}\right\}$ is multiplicatively closed.

Let us consider $R$. One can check that there exists a non empty subset of $R$ which is multiplicatively closed.

Let us consider $A$. Let $V$ be a subset of $A$. We say that $V$ is without zero if and only if

(Def. 4) $0_{A} \notin V$.

Let us observe that there exists a non empty, multiplicatively closed subset of $A$ which is without zero.

Now we state the propositions:

(6) $\Omega_{A} \backslash \mathfrak{p}$ is multiplicatively closed.

(7) Let us consider a proper ideal $J$ of $A$. Then $\operatorname{multClSet}(J, a)$ is multiplicatively closed.

Let us consider $A$. One can check that $\operatorname{NonZeroDivSet}(A)$ is multiplicatively closed.

Let us consider $R$. The functor $\operatorname{UnitSet}(R)$ yielding a subset of $R$ is defined by the term

(Def. 5) $\{a$, where $a$ is an element of $R: a$ is a unit of $R\}$.

Let us observe that $\operatorname{UnitSet}(R)$ is non empty. 
Now we state the proposition:

(8) If $r_{1} \in \operatorname{UnitSet}(R)$, then $r_{1}$ is right mult-cancelable.

Proof: Consider $r_{2}$ such that $r_{2} \cdot r_{1}=1_{R}$. For every elements $u, v$ of $R$ such that $u \cdot r_{1}=v \cdot r_{1}$ holds $u=v$.

Let us consider $R$. Let $r$ be an element of $R$. Assume $r \in \operatorname{UnitSet}(R)$. The functor $\operatorname{recip}(r)$ yielding an element of $R$ is defined by

(Def. 6) it $r=1_{R}$.

We introduce the notation $r^{-1}$ as a synonym of $\operatorname{recip}(r)$.

Let $u, v$ be elements of $R$. The functor $u / v$ yielding an element of $R$ is defined by the term

(Def. 7) $u \cdot \operatorname{recip}(u)$.

Let us consider a unit $u$ of $R$ and an element $v$ of $R$. Now we state the propositions:

(9) If $f$ inherits ring homomorphism, then $f(u)$ is a unit of $R_{1}$ and $f(u)^{-1}=$ $f\left(u^{-1}\right)$.

(10) If $f$ inherits ring homomorphism, then $f\left(v \cdot\left(u^{-1}\right)\right)=f(v) \cdot\left(f(u)^{-1}\right)$. The theorem is a consequence of (9).

\section{Equivalence Relation of Fractions}

In the sequel $S$ denotes a non empty, multiplicatively closed subset of $R$.

Let us consider $R$ and $S$. The functor $\operatorname{Frac}(S)$ yielding a subset of (the carrier of $R) \times($ the carrier of $R$ ) is defined by

(Def. 8) for every set $x, x \in$ it iff there exist elements $a, b$ of $R$ such that $x=\langle a$, b) and $b \in S$.

Now we state the proposition:

(11) $\operatorname{Frac}(S)=\Omega_{R} \times S$.

Let us consider $R$ and $S$. Let us observe that $\operatorname{Frac}(S)$ is non empty.

The functor frac1 $(S)$ yielding a function from $R$ into $\operatorname{Frac}(S)$ is defined by

(Def. 9) for every object $o$ such that $o \in$ the carrier of $R$ holds $i t(o)=\left\langle o, 1_{R}\right\rangle$.

From now on $u, v, w, x, y, z$ denote elements of $\operatorname{Frac}(S)$.

Let us consider $R$ and $S$. Let $u, v$ be elements of $\operatorname{Frac}(S)$. The functor $\operatorname{FracAdd}(u, v)$ yielding an element of $\operatorname{Frac}(S)$ is defined by the term

(Def. 10) $\left\langle(u)_{\mathbf{1}} \cdot(v)_{\mathbf{2}}+(v)_{\mathbf{1}} \cdot(u)_{\mathbf{2}},(u)_{\mathbf{2}} \cdot(v)_{\mathbf{2}}\right\rangle$.

One can verify that the functor is commutative.

The functor FracMult $(u, v)$ yielding an element of $\operatorname{Frac}(S)$ is defined by the term 
(Def. 11) $\left\langle(u)_{\mathbf{1}} \cdot(v)_{\mathbf{1}},(u)_{\mathbf{2}} \cdot(v)_{\mathbf{2}}\right\rangle$.

One can check that the functor is commutative.

Let us consider $x$ and $y$. The functors: $x+y$ and $x \cdot y$ yielding elements of $\operatorname{Frac}(S)$ are defined by terms

(Def. 12) FracAdd $(x, y)$,

(Def. 13) FracMult $(x, y)$,

respectively. Now we state the propositions:

(12) $\operatorname{FracAdd}(x, \operatorname{FracAdd}(y, z))=\operatorname{Frac} A d d(\operatorname{Frac} A d d(x, y), z)$.

(13) $\operatorname{FracMult}(x, \operatorname{FracMult}(y, z))=\operatorname{FracMult}(\operatorname{FracMult}(x, y), z)$.

Let us consider $R$ and $S$. Let $x, y$ be elements of $\operatorname{Frac}(S)$. We say that $x={ }_{F r_{S}} y$ if and only if

(Def. 14) there exists an element $s_{1}$ of $R$ such that $s_{1} \in S$ and $\left((x)_{\mathbf{1}} \cdot\left((y)_{\mathbf{2}}\right)-(y)_{\mathbf{1}}\right.$. $\left.\left((x)_{\mathbf{2}}\right)\right) \cdot s_{1}=0_{R}$.

Now we state the propositions:

(14) If $0_{R} \in S$, then $x={ }_{F r_{S}} y$.

(15) $x=F r_{S} x$.

(16) If $x={ }_{F r_{S}} y$, then $y={ }_{F r_{S}} x$.

(17) If $x={ }_{F r_{S}} y$ and $y={ }_{F r_{S}} z$, then $x={ }_{F r_{S}} z$.

Let us consider $R$ and $S$. The functor $\operatorname{EqRel}(S)$ yielding an equivalence relation of $\operatorname{Frac}(S)$ is defined by

(Def. 15) $\langle u, v\rangle \in$ it iff $u={ }_{F r_{S}} v$.

Now we state the propositions:

(18) $x \in[y]_{\operatorname{EqRel}(S)}$ if and only if $x={ }_{F r_{S}} y$.

(19) $[x]_{\operatorname{EqRel}(S)}=[y]_{\operatorname{EqRel}(S)}$ if and only if $x={ }_{F r_{S}} y$.

Proof: Set $E=\operatorname{EqRel}(S)$. If $[x]_{E}=[y]_{E}$, then $x=_{F r_{S}} y . x \in[y]_{E}$.

(20) If $x={ }_{F r_{S}} u$ and $y={ }_{F r_{S}} v$, then FracMult $(x, y)={ }_{F r_{S}} \operatorname{FracMult}(u, v)$.

(21) If $x={ }_{F r_{S}} u$ and $y={ }_{F r_{S}} v$, then $\operatorname{FracAdd}(x, y)={ }_{F r_{S}} \operatorname{FracAdd}(u, v)$.

(22) $(x+y) \cdot z={ }_{F r_{S}} x \cdot z+y \cdot z$.

Let us consider $R$ and $S$. The functors: $0_{R}^{S \times S}$ and $I_{R}^{S \times S}$ yielding elements of $\operatorname{Frac}(S)$ are defined by terms

(Def. 16) $\left\langle 0_{R}, 1_{R}\right\rangle$,

(Def. 17) $\left\langle 1_{R}, 1_{R}\right\rangle$,

respectively. Now we state the proposition:

(23) Let us consider an element $s$ of $S$. If $x=\langle s, s\rangle$, then $x={ }_{F r_{S}} I_{R}^{S \times S}$. 


\section{Construction of Ring of Fractions}

Let us consider $R$ and $S$. The functor $\operatorname{FracRing}(S)$ yielding a strict double loop structure is defined by

(Def. 18) the carrier of $i t=$ Classes $\operatorname{EqRel}(S)$ and $1_{i t}=\left[I_{R}^{S \times S}\right]_{\operatorname{EqRel}(S)}$ and $0_{i t}=$ $\left[0_{R}^{S \times S}\right]_{\operatorname{EqRel}(S)}$ and for every elements $x, y$ of $i t$, there exist elements $a, b$ of $\operatorname{Frac}(S)$ such that $x=[a]_{\operatorname{EqRel}(S)}$ and $y=[b]_{\mathrm{EqRel}(S)}$ and (the addition of $i t)(x, y)=[a+b]_{\operatorname{EqRel}(S)}$ and for every elements $x, y$ of $i t$, there exist elements $a, b$ of $\operatorname{Frac}(S)$ such that $x=[a]_{\operatorname{EqRel}(S)}$ and $y=[b]_{\operatorname{EqRel}(S)}$ and (the multiplication of $i t)(x, y)=[a \cdot b]_{\mathrm{EqRel}(S)}$.

We introduce the notation $S \sim R$ as a synonym of FracRing $(S)$.

One can verify that $S \sim R$ is non empty.

Now we state the proposition:

(24) $0_{R} \in S$ if and only if $S \sim R$ is degenerated. The theorem is a consequence of (19).

In the sequel $a, b, c$ denote elements of $\operatorname{Frac}(S)$ and $x, y, z$ denote elements of $S \sim R$.

Now we state the propositions:

(25) There exists an element $a$ of $\operatorname{Frac}(S)$ such that $x=[a]_{\operatorname{EqRel}(S)}$.

(26) If $x=[a]_{\operatorname{EqRel}(S)}$ and $y=[b]_{\operatorname{EqRel}(S)}$, then $x \cdot y=[a \cdot b]_{\operatorname{EqRel}(S)}$. The theorem is a consequence of (19) and (20).

(27) $x \cdot y=y \cdot x$. The theorem is a consequence of (25) and (26).

(28) If $x=[a]_{\operatorname{EqRel}(S)}$ and $y=[b]_{\mathrm{EqRel}(S)}$, then $x+y=[a+b]_{\mathrm{EqRel}(S)}$. The theorem is a consequence of (19) and (21).

(29) $S \sim R$ is a ring.

PROOF: $x+y=y+x .(x+y)+z=x+(y+z) \cdot x+0_{S \sim R}=x . x$ is right complementable. $(x+y) \cdot z=x \cdot z+y \cdot z \cdot x \cdot(y+z)=x \cdot y+x \cdot z$ and $(y+z) \cdot x=y \cdot x+z \cdot x \cdot(x \cdot y) \cdot z=x \cdot(y \cdot z) \cdot x \cdot\left(1_{S \sim R}\right)=x$ and $1_{S \sim R} \cdot x=x$.

Let us consider $R$ and $S$. One can verify that $S \sim R$ is commutative, Abelian, add-associative, right zeroed, right complementable, associative, well unital, and distributive.

Now we state the proposition:

(30) There exist elements $r_{1}, r_{2}$ of $R$ such that

(i) $r_{2} \in S$, and

(ii) $z=\left[\left\langle r_{1}, r_{2}\right\rangle\right]_{\operatorname{EqRel}(S)}$.

The theorem is a consequence of (25). 
In the sequel $S$ denotes a without zero, non empty, multiplicatively closed subset of $A$.

Let us consider $A$ and $S$. The canonical homomorphism of $S$ into quotient field yielding a function from $A$ into $S \sim A$ is defined by

(Def. 19) for every object $o$ such that $o \in$ the carrier of $A$ holds it $(o)=$ $[(\operatorname{frac} 1(S))(o)]_{\operatorname{EqRel}(S)}$.

Let us observe that the canonical homomorphism of $S$ into quotient field is additive, multiplicative, and unity-preserving.

Now we state the propositions:

(31) Let us consider elements $a, b$ of $A$. Then (the canonical homomorphism of $S$ into quotient field) $(a-b)=$ (the canonical homomorphism of $S$ into quotient field) $(a)$ - (the canonical homomorphism of $S$ into quotient field)(b).

(32) Suppose $0_{A} \notin S$. Then ker the canonical homomorphism of $S$ into quotient field $\subseteq$ ZeroDivSet $(A)$.

Proof: For every $o$ such that $o \in$ ker the canonical homomorphism of $S$ into quotient field holds $o \in \operatorname{ZeroDivSet}(A)$.

(33) Suppose $0_{A} \notin S$ and $A$ is an integral domain. Then

(i) ker the canonical homomorphism of $S$ into quotient field $=\left\{0_{A}\right\}$, and

(ii) the canonical homomorphism of $S$ into quotient field is one-to-one.

Proof: ker the canonical homomorphism of $S$ into quotient field $\subseteq$ ZeroDiv $\operatorname{Set}(A)$. ZeroDivSet $(A)=\left\{0_{A}\right\}$. For every objects $x, y$ such that $x, y \in$ $\operatorname{dom}$ (the canonical homomorphism of $S$ into quotient field) and (the canonical homomorphism of $S$ into quotient field) $(x)=$ (the canonical homomorphism of $S$ into quotient field)(y) holds $x=y$.

\section{Localization in Terms of Prime Ideals}

From now on $\mathfrak{p}$ denotes an element of the spectrum of $A$.

Let us consider $A$ and $\mathfrak{p}$. The functor $\operatorname{Loc}(A, \mathfrak{p})$ yielding a subset of $A$ is defined by the term

(Def. 20) $\Omega_{A} \backslash \mathfrak{p}$.

One can check that $\operatorname{Loc}(A, \mathfrak{p})$ is non empty and $\operatorname{Loc}(A, \mathfrak{p})$ is multiplicatively closed and $\operatorname{Loc}(A, \mathfrak{p})$ is without zero.

The functor $A \sim \mathfrak{p}$ yielding a ring is defined by the term

(Def. 21) $\operatorname{Loc}(A, \mathfrak{p}) \sim A$.

One can verify that $A \sim \mathfrak{p}$ is non degenerated and $A \sim \mathfrak{p}$ is commutative.

The functor LocIdeal $(\mathfrak{p})$ yielding a subset of $\Omega_{A \sim \mathfrak{p}}$ is defined by the term 
(Def. 22) $\{y$, where $y$ is an element of $A \sim \mathfrak{p}:$ there exists an element $a$ of $\operatorname{Frac}(\operatorname{Loc}(A, \mathfrak{p}))$ such that $a \in \mathfrak{p} \times \operatorname{Loc}(A, \mathfrak{p})$ and $\left.y=[a]_{\operatorname{EqRel}(\operatorname{Loc}(A, \mathfrak{p}))}\right\}$.

Observe that LocIdeal( $\mathfrak{p})$ is non empty.

In the sequel $a, m, n$ denote elements of $A \sim \mathfrak{p}$.

Now we state the propositions:

(34) $\operatorname{LocIdeal}(\mathfrak{p})$ is a proper ideal of $A \sim \mathfrak{p}$.

Proof: Reconsider $M=\operatorname{LocIdeal}(\mathfrak{p})$ as a subset of $A \sim \mathfrak{p}$. For every elements $m, n$ of $A \sim \mathfrak{p}$ such that $m, n \in M$ holds $m+n \in M$. For every elements $x, m$ of $A \sim \mathfrak{p}$ such that $m \in M$ holds $x \cdot m \in M$. $M$ is proper by [2, (19)], (19).

(35) Let us consider an object $x$. Suppose $x \in \Omega_{A \sim \mathfrak{p}} \backslash(\operatorname{LocIdeal}(\mathfrak{p}))$. Then $x$ is a unit of $A \sim \mathfrak{p}$. The theorem is a consequence of (25) and (11).

(36) (i) $A \sim \mathfrak{p}$ is local, and

(ii) LocIdeal(p) is a maximal ideal of $A \sim \mathfrak{p}$.

Proof: Reconsider $J=\operatorname{LocIdeal}(\mathfrak{p})$ as a proper ideal of $A \sim \mathfrak{p} . A \sim \mathfrak{p}$ is local. $J$ is a maximal ideal of $A \sim \mathfrak{p}$ by [8, (8), (11)], (35).

\section{Universal Property of Ring of Fractions}

From now on $f$ denotes a function from $A$ into $B$.

Now we state the proposition:

(37) Let us consider an element $s$ of $S$. Suppose $f$ inherits ring homomorphism and $f^{\circ} S \subseteq \operatorname{UnitSet}(B)$. Then $f(s)$ is a unit of $B$.

Let us consider $A, B, S$, and $f$. Assume $f$ inherits ring homomorphism and $f^{\circ} S \subseteq \operatorname{UnitSet}(B)$. The functor $\operatorname{UnivMap}(S, f)$ yielding a function from $S \sim A$ into $B$ is defined by

(Def. 23) for every object $x$ such that $x \in$ the carrier of $S \sim A$ there exist elements $a, s$ of $A$ such that $s \in S$ and $x=[\langle a, s\rangle]_{\operatorname{EqRel}(S)}$ and it $(x)=f(a)$. $\left(f(s)^{-1}\right)$.

Now we state the propositions:

(38) If $f$ inherits ring homomorphism and $f^{\circ} S \subseteq \operatorname{UnitSet}(B)$, then $\operatorname{UnivMap}(S, f)$ is additive.

Proof: For every elements $x, y$ of $S \sim A,(\operatorname{UnivMap}(S, f))(x+y)=$ $(\operatorname{UnivMap}(S, f))(x)+(\operatorname{UnivMap}(S, f))(y)$.

(39) If $f$ inherits ring homomorphism and $f^{\circ} S \subseteq \operatorname{UnitSet}(B)$, then $\operatorname{UnivMap}(S, f)$ is multiplicative.

Proof: For every elements $x, y$ of $S \sim A,(\operatorname{UnivMap}(S, f))(x \cdot y)=$ $(\operatorname{UnivMap}(S, f))(x) \cdot(\operatorname{UnivMap}(S, f))(y)$. 
(40) If $f$ inherits ring homomorphism and $f^{\circ} S \subseteq \operatorname{UnitSet}(B)$, then $\operatorname{UnivMap}(S, f)$ is unity-preserving.

Proof: $(\operatorname{UnivMap}(S, f))\left(1_{S \sim A}\right)=1_{B}$.

(41) If $f$ inherits ring homomorphism and $f^{\circ} S \subseteq \operatorname{UnitSet}(B)$, then $\operatorname{UnivMap}(S, f)$ inherits ring homomorphism.

(42) Suppose $f$ inherits ring homomorphism and $f^{\circ} S \subseteq \operatorname{UnitSet}(B)$. Then $f=(\operatorname{UnivMap}(S, f)) \cdot($ the canonical homomorphism of $S$ into quotient field).

Proof: Set $g_{1}=(\operatorname{UnivMap}(S, f)) \cdot$ (the canonical homomorphism of $S$ into quotient field). For every object $x$ such that $x \in \operatorname{dom} f$ holds $f(x)=g_{1}(x)$ by $(19),(37),[5,(8)]$.

\section{The Total-Quotient Ring \\ and the Quotient Field of Integral Domain}

Let us consider $A$. The functor TotalQuotRing $(A)$ yielding a ring is defined by the term

(Def. 24) NonZeroDivSet $(A) \sim A$.

Observe that TotalQuotRing $(A)$ is non degenerated.

In the sequel $x$ denotes an object.

Now we state the proposition:

(43) If $A$ is a field, then Ideals $A=\left\{\left\{0_{A}\right\}\right.$, the carrier of $\left.A\right\}$.

Proof: If $x \in$ Ideals $A$, then $x \in\left\{\left\{0_{A}\right\}\right.$, the carrier of $\left.A\right\}$.

If $x \in\left\{\left\{0_{A}\right\}\right.$, the carrier of $\left.A\right\}$, then $x \in$ Ideals $A$.

From now on $A$ denotes an integral domain.

(i) NonZeroDivSet $(A)=\Omega_{A} \backslash\left\{0_{A}\right\}$, and

(ii) NonZeroDivSet $(A)$ is a without zero, non empty, multiplicatively closed subset of $A$.

The theorem is a consequence of (4).

(45) Let us consider an element $a$ of $A$. Then $a \in \operatorname{NonZeroDivSet}(A)$ if and only if $a \neq 0_{A}$. The theorem is a consequence of (44).

(46) TotalQuotRing $(A)$ is a field. The theorem is a consequence of (4), (30), and (19).

(47) Let us consider an integral domain $A$. Then the field of quotients of $A$ is ring isomorphic to TotalQuotRing $(A)$.

Proof: Set $S=$ NonZeroDivSet $(A)$. Set $B=$ the field of quotients of $A$. Set $f=$ the canonical homomorphism of $A$ into quotient field. $f^{\circ} S \subseteq$ $\operatorname{UnitSet}(B)$. Reconsider $S=\operatorname{NonZeroDivSet}(A)$ as a without zero, non 
empty, multiplicatively closed subset of $A$. UnivMap $(S, f)$ inherits ring homomorphism. TotalQuotRing $(A)$ is a field. Set $g=\operatorname{UnivMap}(S, f)$. For every object $y$ such that $y \in \Omega_{B}$ holds $y \in \operatorname{rng} g$.

\section{REFERENCES}

[1] Michael Francis Atiyah and Ian Grant Macdonald. Introduction to Commutative Algebra, volume 2. Addison-Wesley Reading, 1969.

[2] Jonathan Backer, Piotr Rudnicki, and Christoph Schwarzweller. Ring ideals Formalized Mathematics, 9(3):565-582, 2001.

[3] Grzegorz Bancerek, Czesław Byliński, Adam Grabowski, Artur Korniłowicz, Roman Matuszewski, Adam Naumowicz, Karol Pąk, and Josef Urban. Mizar: State-of-the-art and beyond. In Manfred Kerber, Jacques Carette, Cezary Kaliszyk, Florian Rabe, and Volker Sorge, editors, Intelligent Computer Mathematics, volume 9150 of Lecture Notes in Computer Science, pages 261-279. Springer International Publishing, 2015. ISBN 978-3319-20614-1. doi 10.1007/978-3-319-20615-8_17.

[4] Grzegorz Bancerek, Czesław Byliński, Adam Grabowski, Artur Korniłowicz, Roman Matuszewski, Adam Naumowicz, and Karol Pąk. The role of the Mizar Mathematical Library for interactive proof development in Mizar. Journal of Automated Reasoning, 61(1):9-32, 2018. do1 $10.1007 / \mathrm{s} 10817-017-9440-6$

[5] Artur Korniłowicz and Christoph Schwarzweller. The first isomorphism theorem and other properties of rings. Formalized Mathematics, 22(4):291-301, 2014. doi 10.2478/forma-20140029 .

[6] Hideyuki Matsumura. Commutative Ring Theory. Cambridge Studies in Advanced Mathematics. Cambridge University Press, 2nd edition, 1989.

[7] Christoph Schwarzweller. The field of quotients over an integral domain. Formalized Mathematics, 7(1):69-79, 1998.

[8] Yasushige Watase. Zariski topology. Formalized Mathematics, 26(4):277-283, 2018. doi: $10.2478 /$ forma-2018-0024. 